\title{
Arteria mediana persistente: una variación anatómica vascular en el miembro superior. Estudio anatómico, revisión bibliográfica y estudio clínico en 128 pacientes \\ Persistent median artery: a vascular anatomical variation in the upper limb. Anatomical study, bibliographic review and clinical study in 128 patients
}

\author{
María Jesús RIVERA VEGAS*, María Elena RUIZ ALONSO* \\ Paula MIGUELEZ SIERRA**
}

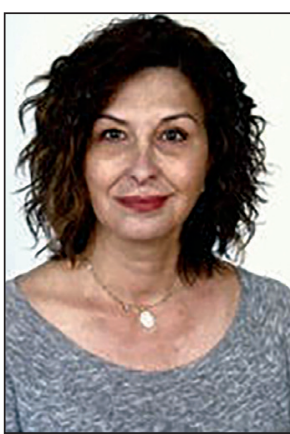

Rivera Vegas M. J.
Introducción y objetivo. En nuestra práctica diaria encontramos variaciones anatómicas que tienen repercusión tanto diagnóstica como terapéutica.

Con este artículo ponemos el acento en la importancia de las variaciones anatómicas vasculares, presentando un caso anatómico de arteria mediana persistente que altera la vascularización de los dedos de la mano y que frecuentemente se asocia a la presencia de un nervio mediano bífido. Tras este hallazgo, realizamos una revisión bibliográfica y un estudio clínico para poner en relieve la asociación de esta malformación con el síndrome del túnel carpiano (STC).

Material y método. Dividimos nuestro estudio en 2 partes: una anatómica y otra clínica.

El estudio anatómico de un antebrazo presentó como hallazgo una arteria mediana palmar persistente responsable de la vascularización del $1^{\text {er }}$ y $2^{\circ}$ dedos de la mano, asociada a un nervio mediano bífido.

Continuamos con una revisión bibliográfica y un estudio prospectivo para conocer el porcentaje de arterias medianas encontradas en la cirugía del STC en pacientes escogidos de manera aleatoria en función de su incorporación a la lista de espera de nuestro hospital durante un periodo de 4 meses.

Resultados. Fueron intervenidos 128 pacientes de STC encontrando arteria mediana persistente en 3 casos, lo que corresponde a un $2.34 \%$ del total valorado.

Discusión. El porcentaje de presentación de arteria mediana persistente en nuestro estudio coincide con los diversos estudios revisados en adultos. Coincidimos con ellos en la importancia del conocimiento de las variaciones anatómicas por si mismas y de su implicación en la sintomatología del STC. También es importante tenerlas en cuenta cuando nos enfrentamos a una lesión traumática $o$ cuando disecamos un colgajo.

Palabras clave Arteria mediana persistente, Síndrome túnel carpiano, Nervio mediano, Cirugía mano

\section{Nivel de evidencia científica Recibido [esta versión] \\ Aceptado}

$5 c$ Terapéutico

$11 \mathrm{abril} / 2019$
24 noviembre/2019
Background and objective. In our daily practice we find anatomical variations that will have a diagnostic and therapeutic repercussion.

With this article we emphasize the importance of vascular anatomical variations presenting an anatomical case of a persistent median artery which alters the vascularization of the fingers, and that is frequently associated with the presence of a bifid median nerve. Following this finding, a literature review and a clinical study were conducted to highlight the association of this malformation with carpal tunnel syndrome (STC)

Methods. Our study is divided into two parts: anatomical and clinical.

The anatomical study of a forearm showed us as a persistent median palmar artery which is responsible for vascularization of the $1^{\text {st }}$ and $2^{\text {nd }}$ fingers, associated with a bifid median nerve.

Then, we conducted a literature review and a prospective study in order to know the percentage of medium arteries found in the STC surgery, chosen in a random way based on their incorporation to the waiting list of our hospital for a 4 months period.

Results. A total of 128 STC patients were operated, finding a persistent median artery in 3 patients, which corresponds to $2.34 \%$ of the total valued.

Discussion. The percentage of presentation of persistent medium artery in our study is coincident with the various studies reviewed in adult patients. We agree on the importance of the knowledge of anatomical variations by themselves and their implication in the symptomatology of STC. It is also important to keep in mind the existence of these anatomical variations when faced with a traumatic injury or when we dissect a flap.

Key words Persistent median artery, Carpal tunnel syndrome, Median nerve, Hand surgery

\section{Level of evidence Received [this version] Accepted}

$5 c$ Therapeutic 22 April/2019

Conflicto de intereses: Los autores declaran no tener ningún interés financiero relacionado con el contenido de este artículo. Financiación: No hubo fuentes externas de financiación para este trabajo. 


\section{Introducción}

La distribución anatómica vascular normal del miembro superior comienza con la arteria axilar como continuación de la arteria subclavia; en el borde inferior del pectoral mayor, esta se convierte en la arteria humeral y en la flexura del codo se bifurca en sus ramas terminales: una externa, la arteria radial y una interna, la arteria cubital. Algunos autores describen el tronco cúbito-interóseo como la porción cubital entre su origen y el nacimiento de las arterias interóseas. El tronco de las interóseas nace de la parte posterior de la cubital y se divide en 2 ramas: la interósea anterior y la interósea posterior. Las ramas terminales de la arteria cubital y de la radial se unen para formar el arco palmar superficial y el arco palmar profundo.

Los cirujanos que trabajan en el miembro superior deben ser conscientes de que las variaciones anatómicas vasculares existen en un porcentaje que oscila entre un $0.26 \%$ a un $18 \%^{(1)}$ dependiendo de localizaciones, y por lo tanto valorar cómo pueden influir en la aparición de determinadas patologías y cómo modificarán determinadas intervenciones.

La complejidad del desarrollo vascular del miembro superior puede dar lugar a numerosas anomalías, como la ausencia de arterias, alteraciones de los orígenes y cursos o la persistencia de arterias embrionarias. ${ }^{(2)}$ Una de estas últimas anomalías es el objeto de nuestro estudio, la arteria mediana persistente. Las estructuras relacionadas con una arteria mediana persistente también pueden ser anómalas. La presencia de una arteria mediana se asocia con una variación anatómica del nervio mediano, que en un $63 \%$ de los casos, suele ser un nervio mediano bífido. ${ }^{(3)}$

En este artículo queremos llamar la atención sobre una variación anatómica poco frecuente, la arteria mediana persistente y su repercusión en la clínica, especialmente con el síndrome del túnel carpiano (STC) en cuanto a su etiología. También poner el foco en que estas arterias medianas persistentes palmares pueden modificar el modelo de vascularización de la mano con el consiguiente peligro en el caso de cirugía y traumas de cualquier tipo, si no somos conocedores de tal variedad.

\section{Material y método}

\section{Caso anatómico modelo}

En una disección anatómica realizada en el Departamento de Anatomía Patológica del Hospital Universitario de Burgos (España) encontramos, de manera casual, una arteria mediana persistente de tipo palmar, lo cual nos estimuló a realizar una revisión bibliográfica y a diseñar el estudio clínico que describiremos en el siguiente apartado.
Procedemos a realizar la descripción anatómica de nuestros hallazgos.

La arteria interósea común se divide desde su inicio, dirigiéndose la arteria interósea posterior hacia la parte dorsal de la membrana interósea, y da una rama anterior de la cual salen la arteria interósea anterior y la arteria mediana persistente (Fig. 1), unida desde su origen al nervio mediano con el que desciende cubierto por la masa muscular de los flexores superficiales (Fig. 2) y apoyado sobre los flexores profundos y el flexor pollicis longus (Fig. 3) y siguiendo las mismas relaciones que el nervio mediano. Además de pequeñas ramas vasculares para los vientres musculares con los que se relaciona, da una rama importante en anchura $(1.2 \mathrm{~mm})$ que une este vaso con la arteria radial previa al ligamento anular del carpo (Fig. 3). Después, atraviesa el ligamento anular del carpo por su parte más radial terminando en 2 arterias comisurales sin unión con las ramas dependientes de la arteria cubital (Fig. 4).

De la rama más radial da una rama para el $1^{\text {er }}$ dedo, que se divide en rama cubital y radial en este y continúa para dar vascularización al borde radial del $2^{\circ}$ dedo. La rama más cubital vasculariza el borde cubital del $2^{\circ}$ dedo y el borde radial del $3^{\text {er }}$ dedo (Fig. 5 y 6 ).

Al completar la disección y levantar los tendones flexores profundos con la exposición de los músculos interóseos palmares, se observa el arco palmar profundo formado por la arteria radial y la rama profunda de la arteria cubital. De este salen unas ramas que se anastomosan con la arteria comisural más radial que el $1^{\text {er }}$ dedo y el borde radial del $2^{\circ}$ dedo.

A la presencia de la arteria mediana persistente se asocia la presencia de un nervio mediano bífido. Este se divide a $5 \mathrm{~cm}$ de la porción más proximal del pecto radial de la eminencia tenar, lo que junto con la alteración vascular antes descrita, motivó nuestro interés por realizar un estudio clínico al respecto.

El tamaño de la arteria mediana en nuestra disección es a su origen de $1.8 \mathrm{~mm}$, que se mantiene hasta llegar al ligamento anular del carpo donde disminuye ligeramente hasta $1.2 \mathrm{~mm}$, tamaño que se mantiene cuando da las comisurales (medición hecha con calibre digital).

\section{Revisión bibliográfica}

Empleamos las palabras clave: persistent median artery, bifid median nerve, persistent median artery and carpal tunnel y llevamos a cabo búsqueda bibliográfica en PubMed, Clinicalkey y Scielo.

\section{Estudio clínico}

Debido a nuestro hallazgo clínico decidimos realizar un estudio prospectivo para estudiar la proporción 


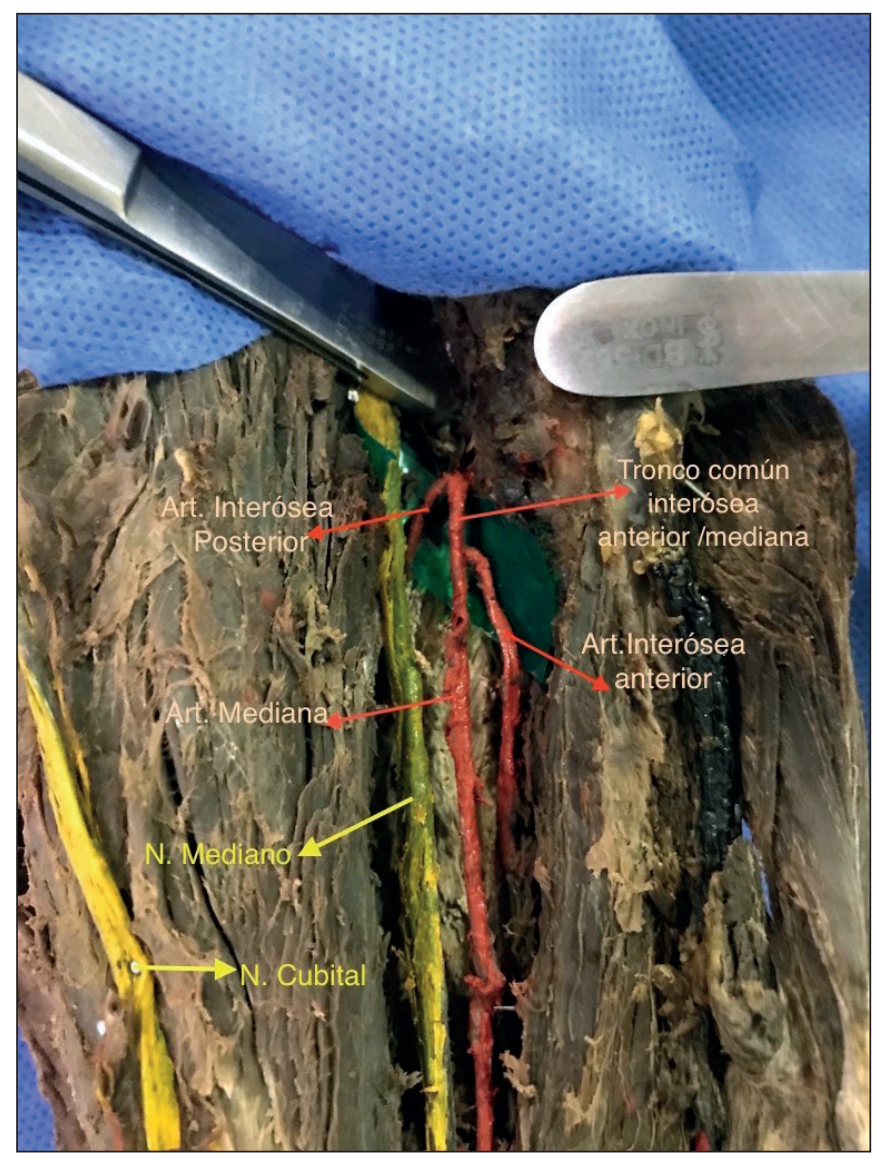

Figura 1. Salida de la arteria interósea común que inmediatamente se divide en la arteria interósea posterior y un tronco común en el que nacen la arteria interósea anterior y la arteria mediana, que se sitúa paralela al nervio mediano junto al cual desciende por el antebrazo.

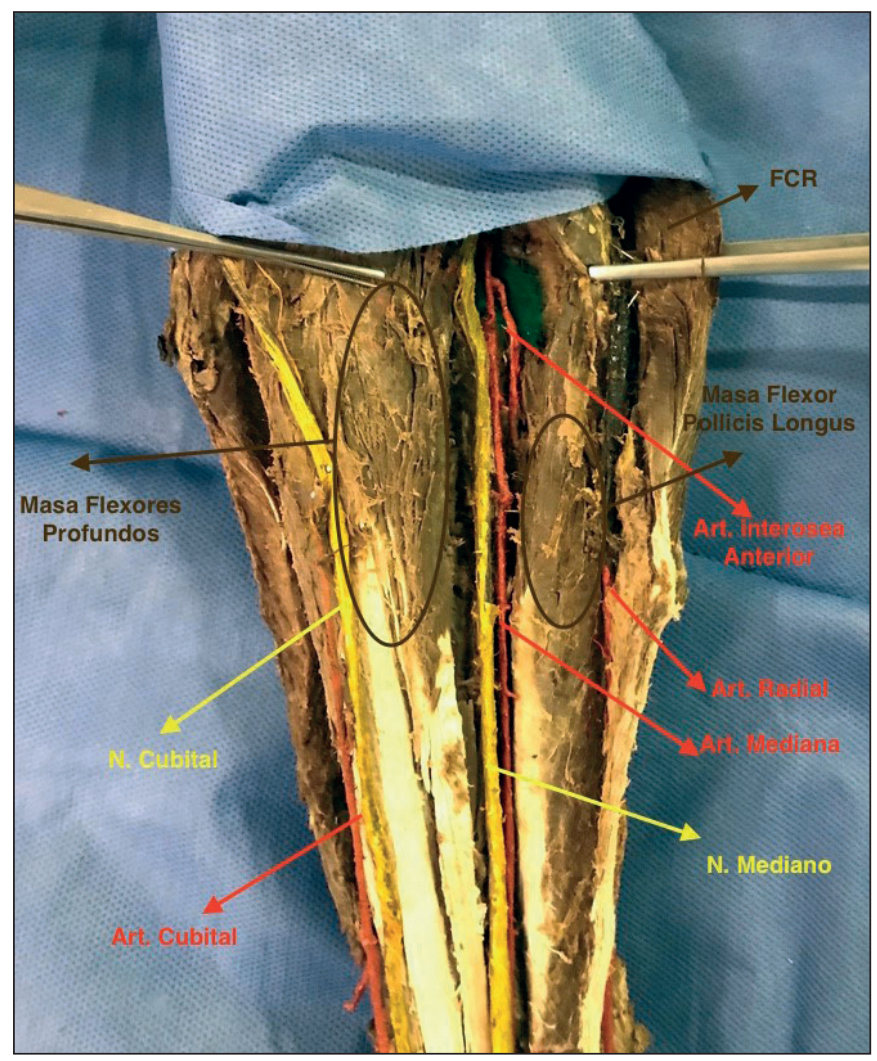

Figura 3. Tercio superior-medio del antebrazo en el que se observa la arteria mediana discurriendo paralela al nervio mediano apoyándose sobre la musculatura flexora profunda.

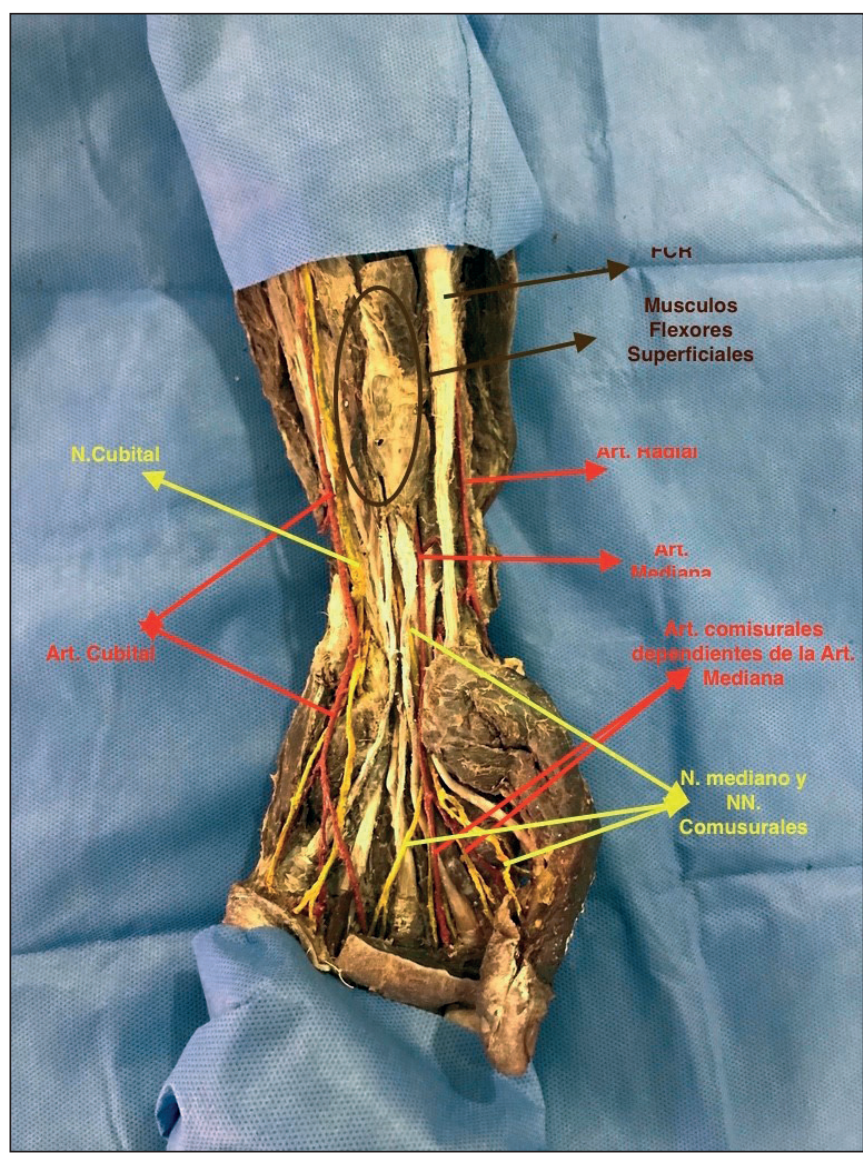

Figura 2. Antebrazo en el que se ha mantenido la musculatura flexora superficial, observando la entrada del nervio mediano y la arteria mediana que dan lugar a las arterias y nervios comisurales en la palma de la mano.

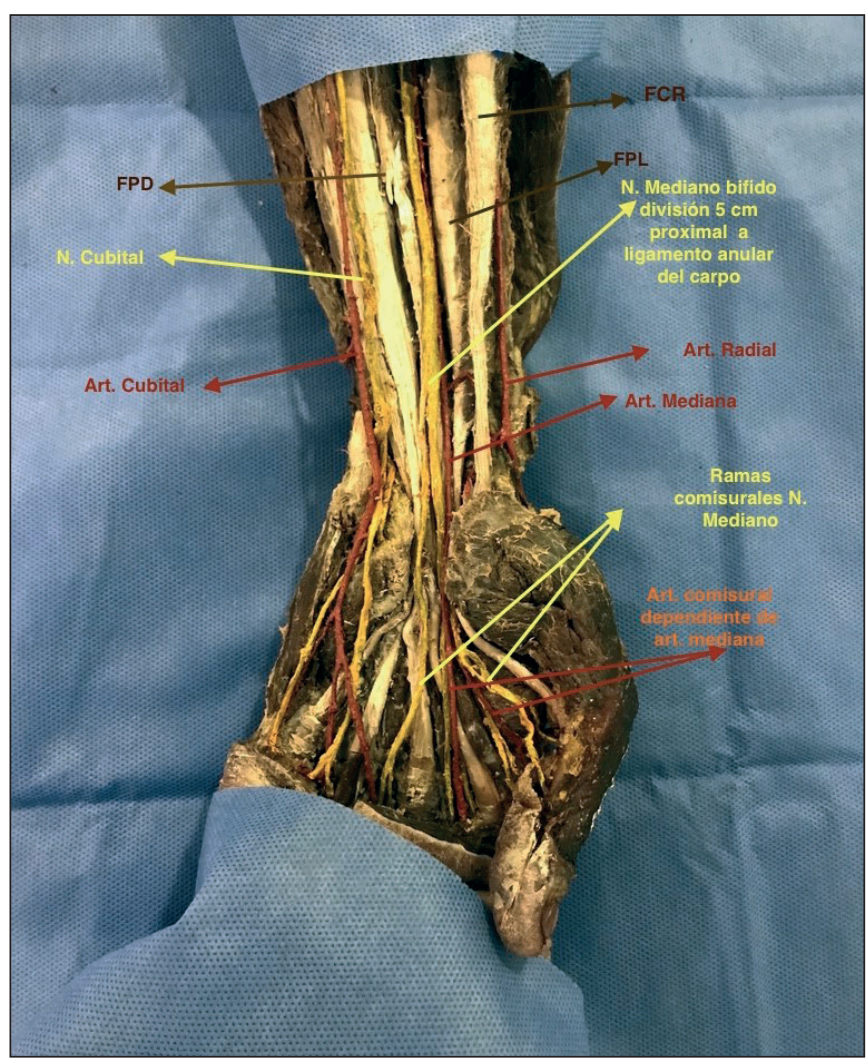

Figura 4. Antebrazo en el que se ha retirado la musculatura de los flexores superficiales observándose el nervio mediano que antes del ligamento anular del carpo, $5 \mathrm{~cm}$., se divide en dos (nervio mediano bífido) acompañado de la arteria mediana persistente. Se observa la rama comunicante entre arteria mediana y radial. La arteria mediana se divide, tras el paso del ligamento anular del carpo, en dos ramas comisurales. 


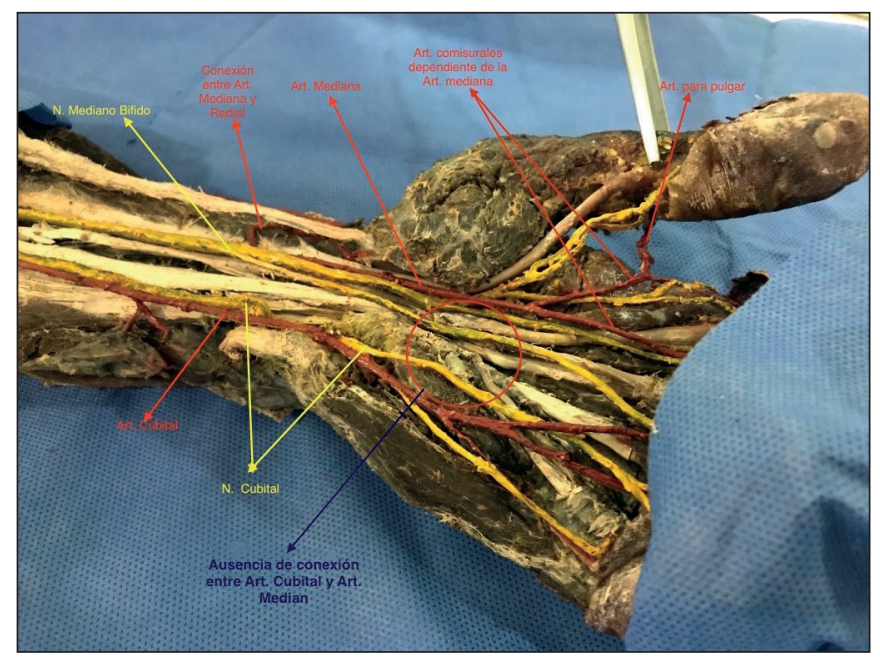

Figura 5. Se observa la ausencia de conexión entre el árbol vascular cubital y el dependiente de la arteria mediana, así como la rama que saliendo de la comisural más radial vasculariza el pulgar.

de arterias medianas palmares persistentes existente en los pacientes intervenidos por STC en nuestro Servicio de Cirugía Plástica del Hospital Universitario de Burgos (España) durante un periodo de 4 meses. Recogimos un total de 128 pacientes seleccionados al azar según su orden de inclusión en la lista de espera quirúrgica de nuestro centro.

Durante los cuatro meses que duró el estudio se mantuvieron las condiciones de realización de la cirugía de apertura del ligamento anular del carpo seguidas habitualmente por nuestro Servicio. La cirugía se realiza bajo anestesia local y apertura del ligamento anular del carpo mediante incisión palmar corta. En los casos en los que se vio una arteria mediana se midió su grosor mediante calibre digital.

\section{Resultados}

De los 128 pacientes intervenidos durante los 4 meses que duró el estudio, 98 fueron mujeres $(76.5 \%)$ de edades comprendidas entre los 30 y los 82 años (media de 56 años) y 30 varones (23.4\%) con edades comprendidas entre los 41 y los 85 años (media de 62 años) (Tabla I).

Todos ellos habían sido incluidos en la lista de espera para tratamiento quirúrgico por STC por los distintos miembros del Servicio, por lo que consideramos que cumplían los requisitos clínicos y las pruebas complementarias para ello.

En la imagen clínica de la Fig. 7 vemos una arteria mediana persistente palmar que es un hallazgo tras la apertura del ligamento anular del carpo por un síndrome de túnel del carpo; vemos una arteria de un calibre de $1,5 \mathrm{~mm}$ sobre el nervio mediano, correspondiente al caso $\mathrm{n}^{\circ} 2$, en el que no se observa la existencia de nervio mediano bífido.

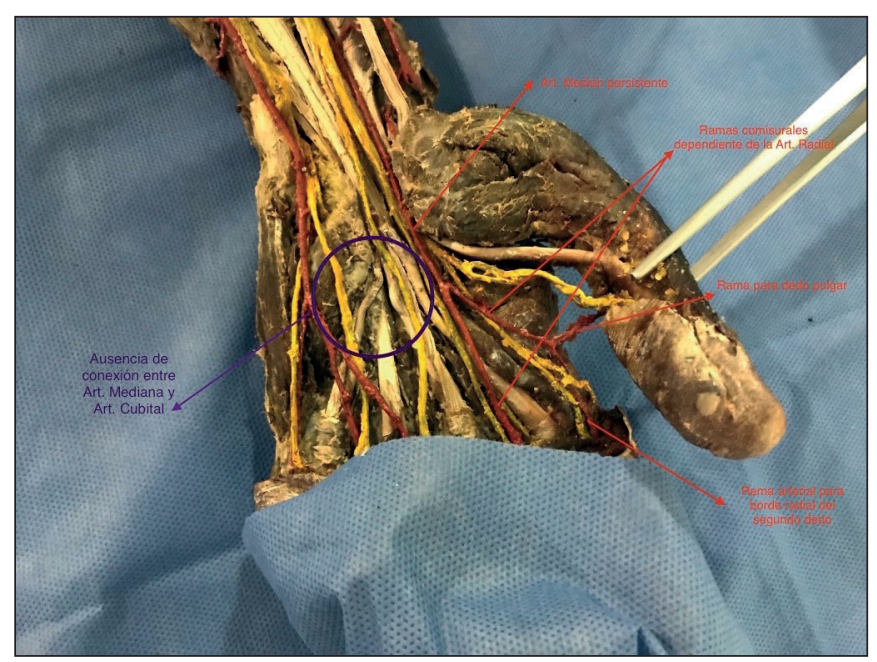

Figura 6. Observamos la rama arterial del borde radial del $2^{\circ}$ dedo, que es rama terminal de la comisural radial.

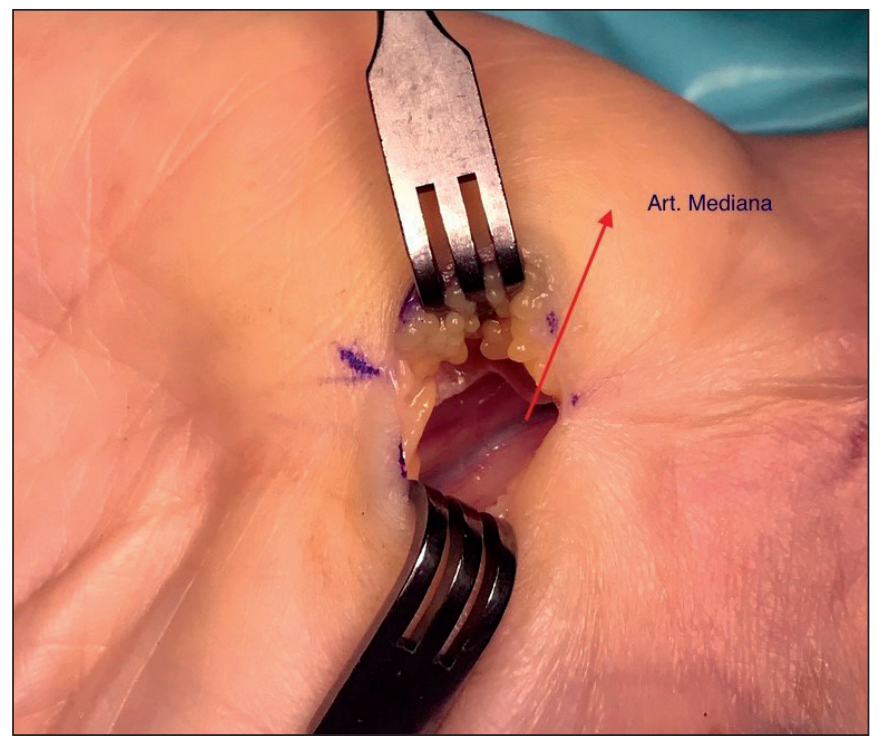

Figura 7. Imagen clínica de apertura de túnel carpiano del caso $n^{\circ} 2$. Se observa una arteria mediana palmar de un tamaño clínico de $1.5 \mathrm{~mm}$.

Encontramos un total de 3 arterias medianas persistentes, lo que supone un $2.34 \%$ de persistencia. Presentamos la información de estos 3 pacientes en la Tabla II.

En nuestro estudio no encontramos ningún nervio bífido, si bien en ningún momento llevamos a cabo actuación sobre el nervio mediano. No se realizó neurolisis con el fin de preservar la estructura nerviosa.

Tabla I. Características de los pacientes de nuestro estudio

\begin{tabular}{|c|c|c|}
\hline \multicolumn{3}{|c|}{ Pacientes intervenidos por } \\
síndrome del túnel carpiano
\end{tabular}


Tabla II. Casos en los que se halló arteria mediana palmar persistente con los datos complementarios correspondientes

\begin{tabular}{|c|c|c|l|c|c|}
\hline Pacientes & $\begin{array}{c}\text { Antecedentes } \\
\text { personales }\end{array}$ & $\begin{array}{c}\text { Tamaño arteria } \\
\text { mediana }\end{array}$ & $\begin{array}{c}\text { Nervio } \\
\text { medianobífido }\end{array}$ & \multicolumn{1}{|c|}{ EMG* } & Sintomatología \\
\hline Caso 1 & Artritis reumatoide & $1.2 \mathrm{~mm}$ & $\begin{array}{l}\text { NO } \\
\text { No neurolisis }\end{array}$ & $\begin{array}{c}\text { Leve } \\
\text { Moderado }\end{array}$ & $\begin{array}{c}\text { Dolor nocturno }+++ \\
\text { Phalen }+++\end{array}$ \\
\hline Caso 2 & Hipotiroidismo & $1.5 \mathrm{~mm}$ & $\begin{array}{l}\text { NO } \\
\text { No neurolisis }\end{array}$ & $\begin{array}{c}\text { Leve } \\
\text { Moderado }\end{array}$ & $\begin{array}{c}\text { Dolor nocturno }+++ \\
\text { Phalen }+++\end{array}$ \\
\hline Caso 3 & Sin interés & $1.5 \mathrm{~mm}$ & $\begin{array}{l}\text { NO } \\
\text { No neurolisis }\end{array}$ & Moderado & $\begin{array}{c}\text { Dolor nocturno }++ \\
\text { Phalen }+\end{array}$ \\
\hline
\end{tabular}

${ }^{*} E M G=$ Electromiograma

\section{Discusión}

Ponemos nuestra atención sobre las variaciones anatómicas vasculares en el miembro superior, y concretamente, en la arteria mediana palmar persistente. Aunque es una variación poco frecuente, su conocimiento es importante y poco recordado, teniendo importancia desde el punto de vista diagnostico y terapéutico por su relación con el STC y alteraciones en el modelo de la vascularización digital.

La arteria mediana es una arteria transitoria que representa el eje arterial del antebrazo durante la vida embrionaria, y que normalmente regresa en el $2^{\circ}$ mes del desarrollo embrionario.

Una hipótesis reciente propone que el suministro arterial de la extremidad superior se desarrolla a partir de un plexo de capilares que experimentan una diferenciación progresiva de proximal a distal entre los días 28-52 de la vida intrauterina, dando como resultado el modelo arterial definitivo. Los vasos sanguíneos en la yema de la extremidad superior proliferan en el mesodermo debido al crecimiento de los vasos preexistentes para formar un plexo vascular. ${ }^{(1)}$ Un componente de este plexo, la arteria axial, forma la arteria braquial en el brazo y continúa como la arteria mediana en el antebrazo. Durante este proceso proliferativo hay una clara sucesión temporal y espacial de la emergencia y la regresión de los vasos, de modo que las variaciones individuales del árbol arterial de la extremidad superior son comunes. ${ }^{(2)}$ Por eso, la variación presente puede ser un remanente del plexo capilar alrededor del nervio mediano que se anastomosa con la arteria interósea anterior mediante procesos de persistencia, agrandamiento y diferenciación de los capilares. Esta hipótesis se enfrenta a la angiogénesis aberrante de vasos, siendo la arteria mediana un caso de persistencia de un vaso sanguíneo.

$\mathrm{Su}$ persistencia en la edad adulta se ha clasificado en 2 modelos diferentes: ${ }^{(3)}$

- Tipo antebraquial: que termina antes de alcanzar la muñeca y se presenta en una proporción mayor entre el 70-100\%.

-Tipo palmar: que alcanza la mano, presentándose en diversos estudios con un porcentaje de entre el 1-5 al 27\%.
A este tipo palmar corresponden las 3 arterias medianas persistentes encontradas en nuestro estudio clínico.

El patrón palmar de la arteria mediana tras el ligamento anular del carpo es variable: la arteria puede terminar en un arco cerrado (tipo mediano-ulnar o radio-mediano-ulnar) o en uno incompleto. En este último patrón, la arteria continúa como la $2^{\mathrm{a}}$ arteria palmar digital común o se divide en dos arterias digitales palmares comunes, dato que es coincidente con nuestro caso de estudio anatómico. El arco palmar superficial incompleto está presente en un $65 \%$ de los casos de persistencia de arteria mediana, mientras que cuando el arco palmar superficial se forma mediante la arteria radial y cubital, solo en un $10-20 \%$ es incompleto. ${ }^{(4)}$ En nuestro estudio, dado que los pacientes fueron intervenidos todos por STC, el estudio de la arteria mediana distal al ligamento anular del carpo estuvo fuera de nuestro alcance.

La presencia de una arteria mediana persistente puede dar lugar a numerosas complicaciones relacionadas con la compresión del nervio mediano proximal y distal. El STC puede ser causado por una arteria mediana permanente. $^{(5)}$ Existen diversas patologías generales como mixedema, artritis reumatoide o el embarazo que pueden predisponer a un STC. En nuestros casos clínicos, 1 de nuestros pacientes presentó como antecedente personal artritis reumatoide y otro, alteraciones tiroideas en tratamiento medico. Cuando estas patologías se asocian a una arteria mediana persistente de más de $1.5 \mathrm{~mm}$, es muy frecuente la presencia de sintomatología de dicho síndrome. También los síntomas de compresión del nervio mediano pueden aparecer cuando se producen alteraciones patológicas de la arteria, como calcificación, ${ }^{(6)}$ trombosis, ${ }^{(7)}$ aterosclerosis, trauma $^{(8)} \mathrm{y}$ dilatación.

Presentamos la imagen ecográfica (Fig. 8) de una paciente pendiente de ser intervenida quirúrgicamente en la que se observa una arteria mediana persistente asociada a un nervio mediano bífido que se localiza entre los dos fascículos. Aunque la paciente presenta síntomas de STC, no presenta signos ecográficos que confirmen 
la sospecha clínica, ya que el nervio mediano bífido presenta un grosor máximo de $8 \mathrm{~mm}$, conservando un patrón fascicular y un grosor normal. Esto apoyaría que un paciente con sintomatología importante y electromiograma leve que no se corresponde con sus síntomas, podría explicarse por la existencia de una arteria mediana persistente. De la misma manera, podríamos explicar los síntomas que se dan en nuestro caso clínico 1 , con electromiograma leve-moderado y sintomatología importante, que decidió su intervención quirúrgica.

La arteria mediana persistente está involucrada en la compresión de los nervios medianos en el antebrazo proximal y el nervio interóseo anterior, lo que resulta en un síndrome de pronador y un síndrome nervio interóseo anterior respectivamente..$^{(9,10)}$

La persistencia de la arteria mediana también puede ocurrir junto con anomalías del nervio mediano. Varios autores han descrito división del nervio mediano por la arteria mediana ${ }^{(11)}$ mientras que otros han informado de la aparición de arteria mediana persistente asociada con una división alta del nervio, observándose una probabilidad más elevada de coexistencia de ambas anomalías (63\%), que la probabilidad de presentarse independientemente. ${ }^{(12)}$ Así ocurre en nuestro caso anatómico, en el que observamos un nervio mediano bífido dividido $5 \mathrm{~cm}$. proximal al ligamento anular del carpo; pero en nuestra serie clínica, con una incisión palmar corta para la apertura del ligamento anular del carpo y sin deseos de actuar quirúrgicamente sobre el nervio con el fin de no producir alteraciones secundarias a una manipulación quirúrgica no necesaria, no pudimos observar la existencia de nervio mediano bífido.

Hemos hecho una revisión bibliográfica de las series de más de 50 pacientes en las que estudian la arteria mediana persistente dividiendo el total en 3 grupos según la diferente población estudiada: a) pacientes intervenidos

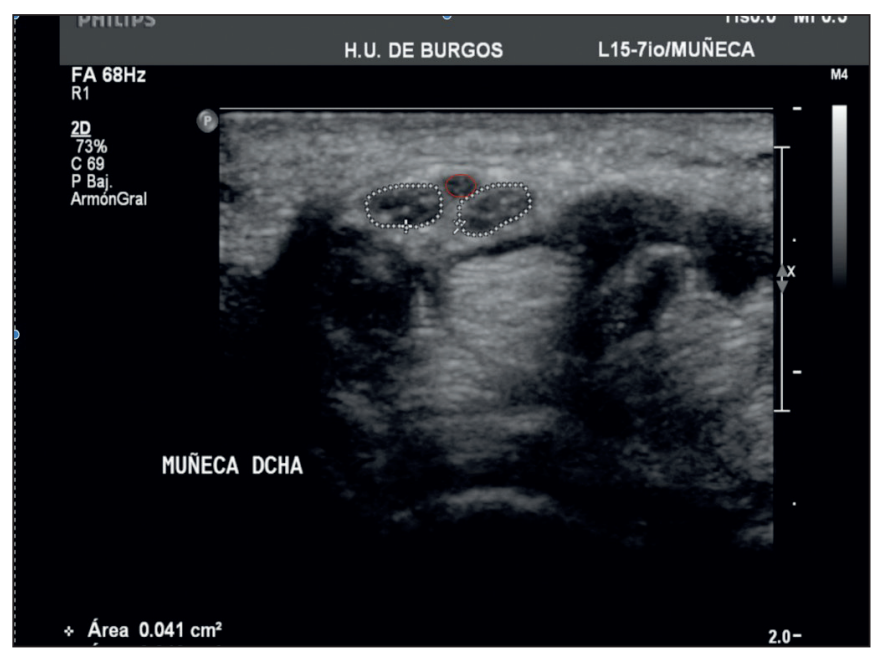

Figura 8. Imagen ecográfica en la que se observa un nervio mediano bífido (imágenes punteadas en blanco) asociado a arteria mediana persistente (imagen limitada en rojo). de STC, como en nuestra serie; b) piezas de cadáveres de adultos; y c) piezas de cadáveres de neonatos y fetos. La incidencia de la arteria mediana persistente presentó grandes variaciones (desde el 0.6 al 44.2\%).

En los artículos de pacientes intervenidos por STC se valora un total de 2.376 pacientes, observando un porcentaje medio de aparición de arteria mediana persistente del $2.18 \%{ }^{(13,14)}$ Nuestro estudio clínico, realizado en 128 pacientes adultos intervenidos por STC, observamos un $2.34 \%$ de individuos que presentaban arterias medianas persistentes de tipo palmar, coincidiendo con los estudios revisados.

Los estudios en cadáveres adultos recogen un total de 2.577 piezas en las que se ha encontrado una media del $8.97 \%$ de persistencia de la arteria mediana. ${ }^{(15,16)}$

En los estudios sobre cadáveres de neonatos y fetos, Kopuz $^{(17)}$ presenta la presencia de arteria mediana persistente en un $20 \%$ de cadáveres neonatales. En el estudio de George y Henneberg, ${ }^{(18)}$ llevado a cabo en cadáveres de recién nacidos y preescolares en Sudáfrica, encontramos una persistencia del $44.2 \%$. En este último grupo de publicaciones de estudios realizados sobre preescolares, neonatos y fetos, el total de piezas estudiadas asciende a 610, con una persistencia media de la arteria mediana del $14 \%$. En los datos obtenidos podemos observar un porcentaje mayor en los cadáveres de neonatos y fetos frente a los pacientes intervenidos de STC adultos o en cadáveres adultos, lo cual estaría en consonancia con la hipótesis de que la arteria mediana regresa en una etapa mucho más tardía, durante el período perinatal y la primera infancia, o incluso más tarde según autores.

Según Rodríguez-Niedenfuhr, ${ }^{(3)}$ el origen de la arteria mediana se situaría en el ángulo entre la arteria cubital y el tronco interóseo común en un 59\% de los casos, siendo este el más frecuente. También puede tener su origen en la arteria cubital (17\%), la arteria interósea anterior (14\%), el tronco interóseo común (7\%) y la arteria radial (3\%). En nuestro caso anatómico, la arteria mediana persistente nace de una arteria común con la interósea anterior tras dar la arteria interósea posterior.

Los diámetros externos de las arterias medianas en los estudios revisados variaron entre 1.5 y $2 \mathrm{~mm}$, coincidiendo con el tamaño encontrado en nuestra arteria tanto en el caso anatómico como en los casos clínicos.

Aunque el patrón palmar de persistencia de la arteria mediana es una anormalidad infrecuente, puede contribuir significativamente al suministro del arco palmar superficial y surgir de cualquiera de las arterias del antebrazo. Su asociación con una alta incidencia de arco palmar incompleta sugiere que debemos evitar la ligadura de la arteria mediana persistente para prevenir síntomas isquémicos en dedos, como sucedería en caso 
de lesión en un modelo anatómico igual a nuestro caso anatómico.

Cuando se planea un colgajo radial y realizar un test de Allen, en caso de que exista una arteria mediana persistente, este test puede no provocar alteraciones ya que la vascularización de los dedos más radiales dependería de la arteria mediana. ${ }^{(19)}$ En nuestro estudio anatómico, el arco palmar superficial no existe, es decir no hay relación entre la arteria mediana persistente y la rama superficial del cubital. El arco profundo se forma con una rama terminal de la radial que sobre los metacarpianos se unen formando un arco de tamaño adecuado $(2.2 \mathrm{~mm})$ con la rama profunda de la arteria cubital. En realidad, la realización de un colgajo radial ya sea a flujo directo como colgajo libre o a flujo inverso sería posible, por lo menos desde un punto de vista anatómico.

Sugerimos que el hallazgo de una arteria mediana durante el acto quirúrgico debe alertar al cirujano sobre la posibilidad de anomalías asociadas y riesgo de isquemia.

De todo lo expuesto se deduce la importancia de la ecografía en el estudio del STC, ya que facilita la localización anatómica de posibles anomalías que, como en el caso de una arteria mediana persistente, son poco frecuentes; en este caso, dado nuestro conocimiento, podremos evitar complicaciones importantes para la vascularización de la mano. ${ }^{(20)}$

\section{Conclusiones}

La existencia de variaciones anatómicas debe ser considerada en nuestros diagnósticos y tratamientos, como en el caso de la arteria mediana persistente y su relación con la aparición del síndrome del tunel carpiano, así como de la modificación de la vascularización de los dedos, a fin de valorar que esta circunstancia pueda suponer un peligro para la viabilidad vascular de la extremidad así como para la planificación de colgajos regionales

\section{Dirección del autor}

Dra. María Jesús Rivera Vegas

Servicio de Cirugía Plástica

Hospital Universitario de Burgos

$\mathrm{C} /$ Islas Baleares $\mathrm{n}^{\circ} 3$

09006 Burgos, España

Correo electrónico: riveravegas@gmail.com

Bibliografía

1. Rodríguez-Niedenführ M, Vázquez T, Nearn L, Ferreira B, Parkin I, Sañudo JR. Variations of the arterial pattern in the upper limb revisited: a morphological and statistical study, with a review of the literature. J. Anat. 2001;199(Pt 5):547-566.
2. Rodríguez-Baeza A, Nebot $\mathbf{J}$, Ferreira $\mathbf{B}$, Reina $\mathbf{F}$, Pérez $\mathbf{J}$, Sañudo JR, Roig M. An anatomical study and ontogenetic explanation of 23 cases with variations in the main pattern of the human brachio-antebrachial arteries. J Anat. 1995; 187:473-479.

3. Rodríguez-Niedenführ M, Sanudo JR, Vazquez T, Nearn L, Logan B, Parkin I. Median artery revisited. J Anat 1999;195:5763.

4. Bilge O, Pinar Y, Ozer MA. A morphometric study on the superficial palmar arch of the hand. Surg Radiol Anat 2006; 28(4):343-350.

5. Gassner EM, Schocke M, Peer S, Schwabegger A, Jaschke W, Bodner G. Persistent median artery in the carpal tunnel: Color Doppler ultrasonographic findings. $J$ Ultrasound Med 2002;21:455-461.

6. Dickinson JC, Kleinbert JM. Acute carpal-tunnel syndrome caused by a calcified median artery. A case report. J. Bone Joint Surg. Am. 1991;73:610-611.

7. Kele H, Verheggen R, Reimers CD. Carpal tunnel syndrome caused by thrombosis of the median artery: The importance of high-resolution ultrasonography for diagnosis. Case report. $J$ Neurosurg 2002;97:471-473.

8. Tsagarakis M, Tarabe M, Minoyiannis N, Tserotas P, Komninakis E. Management of traumatic complete laceration of the median artery at the carpal tunnel: Repair or ligate? Plast Reconstr Surg 2004;114:1014-1015.

9. Lee MJ, La Stayo PC. Pronator syndrome and other nerve compressions that mimic carpal tunnel syndrome. J Orthop. Sports Phys. Ther. 2004;34:601-609.

10. Proudman TW, Menz PJ. An anomaly of the median arteryassociated with the anterior interosseous nerve syndrome. J. Hand Surg. 1992;17: 507-509.

11. Chen L, Chen J, Hu B, Jiang LX. Sonographic Findings of the Bifid Median Nerve and Persistent Median Artery in Carpal Tunnel: A Preliminary Study in Chinese Individuals. Clinics (Sao Paulo) 2017;72(6):358-362.

12. Natsis K, Lordache GI, Gigis I, Kyriazidou A, Lazaridis N, Noussios G, Paraskevas G. Persistent median artery in the carpal tunnel: anatomy, embryology, clinical significance, and review of the literature. Folia Morphol. 2009; 68(4):193-200.

13. Bilgin SS, Olcay SE, Derincek A, Adiyaman S, Demirtas AM. Can simple release relieve symptoms of carpal tunnel syndrome caused by a persistent median artery? Clinical experience. Arch Orthop Trauma Surg, 2004;124:154-156.

14. Ahn DS, Yoon ES, Koo SH, Park SH. A prospective study of the anatomic variations of the median nerve in the carpal tunnel in Asians. Ann Plast Surg, 2000;44:282-287.

15. Olave E, Prates JC, Gabrielli C, Pardi P. Median artery and superficial palmar branch of the radial artery in the carpal tunnel. Scand J Plast Reconstr Hand Surg 1997;31:13-16.

16. Libersa Cl, Francke JP, Mauppin JM, Bailleul JP, Gamblin Ph. The arterial supply to the palm of the hand. Clin Anat 1982;4:33-45.

17. Kopuz C, Baris S, Gulman B. A further morphological study of the persistent median artery in neonatal cadavers. Surg Radiol Anat 1997; 19:403-406.

18. George BJ, Henneberg M. High frequency of the median artery of the forearm in South African newborns and infants. S Afr Med $J$ 1996;86: 175-176.

19. Iain V, Craig JW, Lachlan MC. The median artery: Its potential implications for the radial forearm flap. J. Plast. Reconstr. Aesthet. Surg 2008;61:693-695.

20. Altinkaya N, Leblebici B. Prevalence of persistent median artery in carpal tunnel syndrome: sonographic assessment. Surg. Radiol. Anat. 2016;38(4):511-515. 
